\title{
On the On-Off Problem: An Objective Bayesian Analysis
}

\section{Max Ludwig Ahnen*}

ETH Zurich, Institute for Particle Physics, Otto-Stern-Weg 5, 8093 Zurich, Switzerland

E-mail: mahnen@phys.ethz.ch

The On-Off problem, aka. Li-Ma problem, is a statistical problem where a measured rate is the sum of two parts. The first is due to a signal and the second due to a background, both of which are unknown. Mostly frequentist solutions are being used that are only adequate for high count numbers. When the events are rare such an approximation is not good enough. Indeed, in high-energy astrophysics this is often the rule rather than the exception.

I will present a universal objective Bayesian solution that depends only on the initial three parameters of the On-Off problem: the number of events in the "on" region, the number of events in the "off" region, and their ratio-of-exposure.

With a two-step approach it is possible to infer the signal's significance, strength, uncertainty or upper limit in a unified a way. The approach is valid without restrictions for any count number including zero and may be widely applied in particle physics, cosmic-ray physics and high-energy astrophysics. I apply the method to Gamma Ray Burst data.

The 34th International Cosmic Ray Conference,

30 July- 6 August, 2015

The Hague, The Netherlands

${ }^{*}$ Speaker. 


\section{Introduction}

Typical counting experiments measure discrete sets of events. Such data are often $[1,2]$ modeled with the Poisson distribution. The Poisson distribution may be approximated by a normal distribution when measuring many events. However, when data is rare, such an approximation is not good enough. In Fig. 1, a typical example of a low count data sample is shown. In this case it is an observation of a Gamma Ray Burst (GRB) with the Fermi-LAT instrument. The question arises: What do you do when it simply impossible to "go out and get more data"?

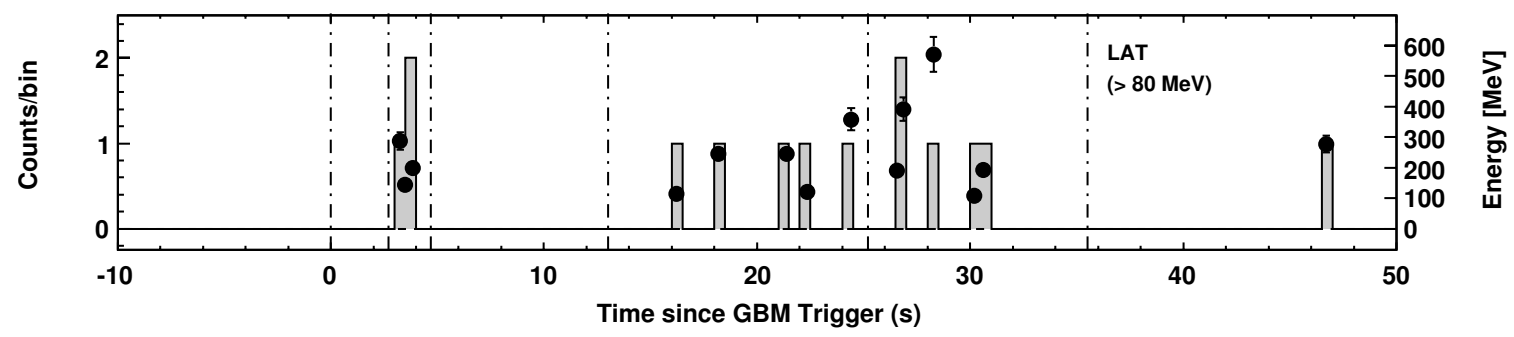

Figure 1: A typical low count high-energy astrophysics data set. It shows gamma rays measured from GRB080825C as observed by Fermi-LAT. Black dots represent the energy measurement, the gray bars represent the number of photons. Figure reproduced from [3]

\section{The On-Off Problem}

In the On-Off problem, also known as Li-Ma problem, one would like to infer a signal rate in the presence of an imprecisely known background rate. The measurement consists of the observation of $N_{\text {on }}$ events in some "on" region with a potential signal and $N_{\text {off }}$ events in some "off" region, known to be signal free. Additionally to the number counts in the on-and off regions, there is a third parameter. This parameter is the ratio $\alpha$ of exposures for the "on" and "off" regions and taken to be known with negligible uncertainty. In the case of gamma ray astronomy, Berge et al. [4] explain the problem and its parameters well.

The common frequentist analyses, based on likelihood ratios and other methods [1,2,5] often assume normal distributed random numbers and therefore lose their foundation when applying them to low count numbers. They also get into trouble at the border of the physical parameter space [5].

The common Bayesian solutions to the On-Off problem are either subjective Bayesian (using proper posteriors [6]) or they avoid specifying the alternative hypothesis at all by using some tail-area probability inference in the spirit of p-values [7]. Subjective Bayesian methods usually introduce a fourth, subjective, parameter (often the upper limit to the signal parameter) which makes the probability statement somewhat dependent on the individual physicist. The tail-area methods, besides ignoring the beauty of Bayesian hypothesis testing, seem to overestimate the probability [6].

Most of all none of the methods in the literature so far cover the full range of the problem: First, the probability that the observed counts are due to background only has to be calculated. Second, the signal contribution has to be estimated. In this proceeding I present a two-step objective 
Bayesian solution to the full On-Off problem [8], inspired by [9], that addresses these issues in a unified a way.

\section{Methods Development}

The idea behind objective Bayesian analysis simple. One takes, in a sense, "flat" priors representing the lack of knowledge. These are usually improper (do not integrate to one). However, in combination with Bayes theorem these can be used to produce proper posteriors and answer a basic what-if question: What is the result if the data were dominant?

One particularly popular objective Bayesian prior is Jeffreys's rule [10, 11]. Jeffreys was motivated by invariance requirements and suggested to take a specific objective prior to make the result (the posterior) invariant under re-parametrization. This prior is a keystone in this analysis.

The analysis follows the method outlined by [9] and is done in two steps. First, the odds that the observed counts are due to the background model are calculated. If this is smaller than a previously defined value, the signal is said to be detected. Second, the signal contribution or upper limit is calculated, depending on whether the detection limit has been reached. The first is done with objective Bayesian hypothesis testing via Bayes factors. The second is done with objective Bayesian estimation.

\subsection{First step: Hypothesis testing}

One problem that appears is that objective priors are only defined up to a proportionality constant and those constants become relevant in this case. A full discussion of the topic can be found in $[8,12]$. In short, there is no generally agreed objective Bayesian hypothesis testing. I suggest to use a method sometimes called "minimal sample device" [13, 14], in order to fix the issue with the proportionality constants. The evaluation of these assumptions in the case of the On-Off problem can be found in $[8,12]$. After all, the odds of the background model over the signal model are

$$
B_{01}=\frac{c_{0}}{c_{1}} \frac{\gamma}{\delta}
$$

where $\gamma$ and $\delta$ are defined using the Gamma function $\Gamma(x)$ and the hypergeometric function ${ }_{2} \mathrm{~F}_{1}(a, b ; c ; z)$ :

$$
\begin{aligned}
\gamma & :=\left(1+2 N_{\text {off }}\right) \alpha^{\frac{1}{2}+N_{\text {on }}+N_{\text {off }}} \Gamma\left(\frac{1}{2}+N_{\text {on }}+N_{\text {off }}\right) \\
\delta & :=2(1+\alpha)^{N_{\text {on }}+N_{\text {off }}} \Gamma\left(1+N_{\text {on }}+N_{\text {off }}\right)_{2} \mathrm{~F}_{1}\left(\frac{1}{2}+N_{\text {off }}, 1+N_{\text {on }}+N_{\text {off }} ; \frac{3}{2}+N_{\text {off }} ;-\frac{1}{\alpha}\right) \\
\frac{c_{0}}{c_{1}} & =\frac{2 \arctan \left(\frac{1}{\sqrt{\alpha}}\right)}{\sqrt{\pi}} .
\end{aligned}
$$

A signal detection based on Eqn. 3.1 may be claimed when the resulting odds of the background model are low. I propose to use use a "Bayesian z-value", similar to [7]

$$
S_{\mathrm{b}}=\sqrt{2} \operatorname{erf}^{-1}\left(1-B_{01}\right)
$$


where $B_{01}=5.710^{-7}$ would correspond to $S_{\mathrm{b}}=5$ or " 5 sigma“. This definition allows for an easy comparison with frequentist significance methods $[8,12]$. However one must keep in mind that the odds of a model and the frequency of an outcome are two different things. $B_{01}$ explicitly weighs alternative models, while the frequentist methods do not.

\subsection{Second Step: Signal Estimation}

After determining the Bayes factor of the background model over the signal model, one proceeds to estimating the signal contribution. This is done via objective Bayesian estimation. If the data show a significant detection the signal model can be assumed to be true. Then, the most probable signal parameter value should be calculated and a physical error interval should be given. If the data show no significant detection, an upper limit on the signal parameter should be calculated, assuming that the signal is there (i.e. the signal model is true) but too weak to be measured. In both cases one needs the conditional probability $P\left(\lambda_{\mathrm{s}} \mid N_{\mathrm{on}}, N_{\mathrm{off}}, H_{1}\right)$ of the signal $\lambda_{\mathrm{s}}$, given the number counts and the signal model $H_{1}$. The improper prior is acceptable in this case because the proportionality constant $c_{1}$ cancels and the posterior is proper. After marginalization over the background parameter $\lambda_{\mathrm{bg}}$, the result is (calculation in [8])

$$
P\left(\lambda_{\mathrm{s}} \mid N_{\mathrm{on}}, N_{\mathrm{off}}, H_{1}\right)=P_{\mathrm{P}}\left(N_{\mathrm{on}}+N_{\text {off }} \mid \lambda_{\mathrm{s}}\right) \frac{\mathrm{U}\left[\frac{1}{2}+N_{\text {off }}, 1+N_{\text {off }}+N_{\mathrm{on}},\left(1+\frac{1}{\alpha}\right) \lambda_{\mathrm{s}}\right]}{{ }_{2} \tilde{\mathrm{F}}_{1}\left(\frac{1}{2}+N_{\text {off }}, 1+N_{\text {off }}+N_{\mathrm{on}} ; \frac{3}{2}+N_{\text {off }} ;-\frac{1}{\alpha}\right)},
$$

as expressed in terms of three functions, namely the Poisson distribution $P_{\mathrm{P}}(N \mid \lambda)$, the regularized hypergeometric function ${ }_{2} \tilde{\mathrm{F}}_{1}(a, b ; c ; z)=\frac{{ }_{2} \mathrm{~F}_{1}(a, b ; c ; z)}{\Gamma(c)}$, and the Tricomi confluent hypergeometric function $\mathrm{U}(a, b, z)$. This posterior contains the full signal parameter information. In order to state a flux, one should take the mode $\lambda_{\mathrm{s}}^{*}$, of the posterior distribution $P\left(\lambda_{\mathrm{s}} \mid N_{\text {on }}, N_{\text {off }}, H_{1}\right)$, as signal estimator. The error on the signal estimator can be evaluated numerically from the cumulative distribution function. On interesting choice is the highest posterior density interval (HPD) $\left[\lambda_{\min }, \lambda_{\max }\right][8]$ containing $68 \%$ probability, calculated as

$$
\int_{\lambda_{\min }}^{\lambda_{\max }} P\left(\lambda_{\mathrm{s}} \mid N_{\mathrm{on}}, N_{\mathrm{off}}, H_{1}\right) d \lambda_{\mathrm{s}}=0.68
$$

together with the constraint

$$
P\left(\lambda_{\min } \mid N_{\text {on }}, N_{\text {off }}, H_{1}\right)=P\left(\lambda_{\max } \mid N_{\text {on }}, N_{\text {off }}, H_{1}\right) .
$$

In case an upper limit should be calculated one can solve the cumulative distribution function, for instance, for a $99 \%$ probability limit $\lambda_{99}$ on the signal parameter $\lambda_{\mathrm{s}}$ as

$$
\int_{0}^{\lambda_{99}} P\left(\lambda_{\mathrm{s}} \mid N_{\mathrm{on}}, N_{\text {off }}, H_{1}\right) d \lambda_{\mathrm{s}}=0.99
$$

These results are natural in a Bayesian approach of the problem but hard to calculate in a frequentist approach. Most frequentist methods particularly struggle with the marginalization and fail at the border of the parameter space. In this approach, all possible number counts are dealt with in a uniform way, no matter if zero counts or thousands of counts. A further benefit is that the signal posterior probability intervals are always physically meaningful (i.e. positive $\lambda_{\mathrm{s}}^{*}, \lambda_{\min }, \lambda_{\max }, \lambda_{99}, \ldots$ ). 
As an application of the developed method, I want to demonstrate the calculations on two measurements of gamma rays from GRBs. These are extraterrestrial flashes of gamma rays mostly lasting only a few seconds. One interesting question is how exactly GRBs produce high energy gamma rays [3]. Because of the GRBs duration and fluence, satellites and Cherenkov telescopes measure only few events during the flare itself or shortly after.

The first example is the GRB080825C as seen by Fermi-LAT (see Sec. 1). In Fig. 1 one sees the light curve in gamma rays $>80 \mathrm{MeV}$. In total there were $N_{\text {on }}=15$ on events and $N_{\text {off }}=19$ off events, with an exposure ratio of $\alpha=33 / 525$ [3]. Running the numbers shows that the odds of the background model over the signal model are (Eqn. 3.1) $B_{01}=9.66 \times 10^{-10}$, or as expressed with the nonlinear scale of Eqn. 3.3, $S_{\mathrm{b}}=6.11$. These numbers compare well to the published value of $S_{\mathrm{li}-\mathrm{ma}}=6.4$ [3]. Clearly, the odds of the background model are low and the GRB is therefore detected. Then, in the second step, one performs the signal estimation. The result is plotted in Fig. 2. The result of $\lambda_{\mathrm{s}}=13.28_{-3.49}^{+4.16}$ is in good agreement with the published reference

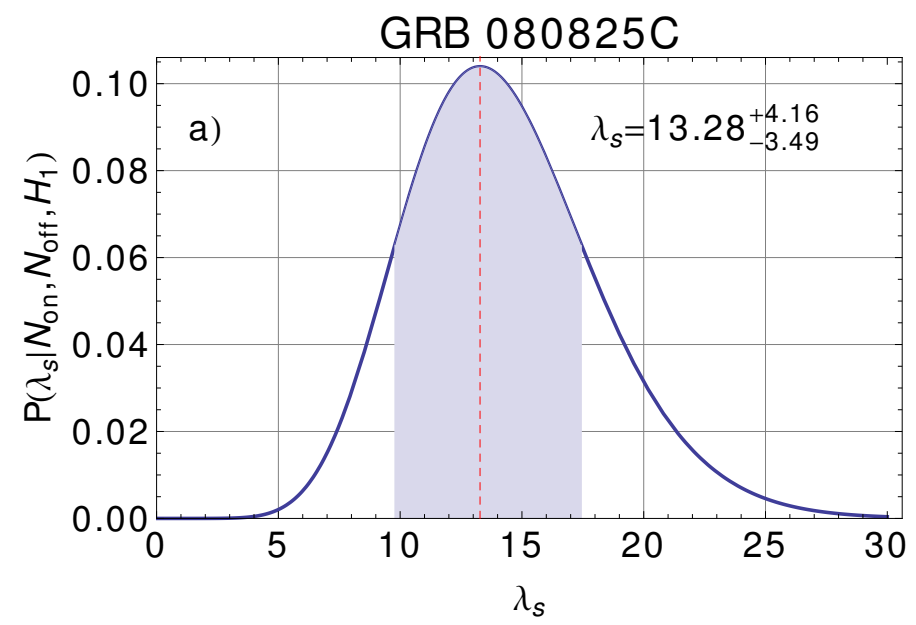

Figure 2: The conditional probability $P\left(\lambda_{\mathrm{s}} \mid N_{\mathrm{on}}, N_{\mathrm{off}}, H_{1}\right)$ of the signal $\lambda_{\mathrm{s}}$, given the Fermi-LAT number counts of GRB080825C. The blue band indicates the HPD interval for the signal parameter posterior probability. Figure reproduced from [8].

of $\lambda_{\text {Ref. }}=13.7$.

The second example is the GRB080330 as observed by the VERITAS Cherenkov telescope [15]. The measurement shows $N_{\text {on }}=0$ on events and $N_{\text {off }}=15$ off events, with an exposure ratio of $\alpha=0.123$ [8]. The corresponding odds of the background model are $B_{01}=2.29$, unsurprisingly favoring the null hypothesis as not a single on event was detected. Now, assuming that the source is there one can put an upper limit to $\lambda_{\mathrm{s}}$. The result is plotted in Fig. 3. The published value uses a frequentist upper limit setting method, popularized by Rolke et al. [5]. Their result is $\lambda_{99}^{\text {Rolke }}=2.4$ [8], which somewhat lower than the number from Eqn. 3.7, $\lambda_{99}=4.10$. A detailed analysis indicates [8] that, especially at the border of the parameter space for $N_{\text {on }} \leq \alpha N_{\text {off }}$, Rolke's method is an overestimation and therefore limited. These limits are overcome by the Bayesian method.

\section{Validation and Discussion}

In order to validate the method and to check if the assumptions made are sensible, an extensive 


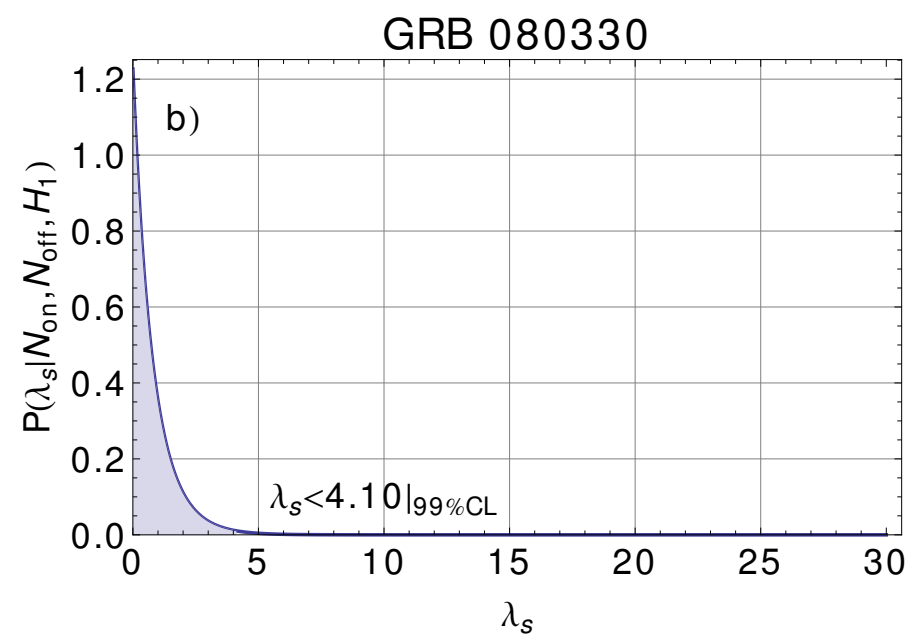

Figure 3: The conditional probability $P\left(\lambda_{\mathrm{s}} \mid N_{\mathrm{on}}, N_{\mathrm{off}}, H_{1}\right)$ of the signal $\lambda_{\mathrm{s}}$, given the VERITAS number counts of GRB080330. The blue band indicates the $99 \%$ probability upper limit to the signal parameter. Figure reproduced from [8].

validation was made [8]. The validation shows that the two-step method behaves well in all testcase examples, in particular at $N_{\text {on }} \sim \alpha N_{\text {off }}$. The objective Bayesian hypothesis testing converges to the results from other methods for high count numbers. The objective Bayesian signal estimation can reconstruct the true signal parameter $\lambda_{\mathrm{s}}$ with a good error estimate.

\section{Conclusion}

Claiming detections, setting credibility intervals, or setting upper limits can be unified over the whole On-Off problem parameter range in one consistent two-step objective Bayesian method. An example implementation in Python can be downloaded from the public git-repository https://bitbucket.org/mknoetig/obayes_onoff_problem.

\section{References}

[1] T. P. Li and Y. Q. Ma, Analysis methods for results in gamma-ray astronomy, Astrophys. J. 272 (1983) 317-324.

[2] R. D. Cousins, J. T. Linnemann, and J. Tucker, Evaluation of three methods for calculating statistical significance when incorporating a systematic uncertainty into a test of the background-only hypothesis for a poisson process, Nucl. Instrum. Methods A 595 (2008), no. 2 480-501.

[3] Fermi LAT/GBM Collaborations Collaboration, A. Abdo et al., Fermi observations of high-energy gamma-ray emission from grb 080825c, Astrophys. J. 707 (2009), no. 1580.

[4] D. Berge, S. Funk, and J. Hinton, Background modelling in very-high-energy $\gamma$-ray astronomy, Astron. Astrophys. 466 (May, 2007) 1219-1229.

[5] W. A. Rolke, A. M. López, and J. Conrad, Limits and confidence intervals in the presence of nuisance parameters, Nucl. Instrum. Methods A 551 (2005), no. 2 493-503. 
[6] P. Gregory, Bayesian logical data analysis for the physical sciences. Cambridge University Press, 2005.

[7] S. Gillessen and H. L. Harney, Significance in gamma-ray astronomy - the Li - Ma problem in Bayesian statistics, Astron. Astrophys. 430 (2005) 355-362.

[8] M. L. Knoetig, Signal discovery, limits, and uncertainties with sparse on/off measurements: an objective bayesian analysis, Astrophys. J. 790 (2014), no. 2106.

[9] A. Caldwell and K. Kröninger, Signal discovery in sparse spectra: A bayesian analysis, Phys. Rev. D 74 (2006), no. 9092003.

[10] H. Jeffreys, Theory of probability. Clarendon Press Oxford, 1961.

[11] Particle Data Group Collaboration, J. Beringer et al., Review of particle physics, Phys. Rev. D 86 (2012) 010001

[12] M. L. Ahnen, On the on/off problem, in Bayes Forum Munich, 2014.

[13] D. J. Spiegelhalter and A. F. M. Smith, Bayes factors for linear and log-linear models with vague prior information, J. R. Statist. Soc. B 44 (1981), no. 3 377-387.

[14] J. K. Ghosh and T. Samanta, Nonsubjective bayes testing - an overview, J. Statist. Plann. Inference 103 (2002) 205-223.

[15] VERITAS Collaboration Collaboration, V. A. Acciari et al., VERITAS Observations of Gamma-Ray Bursts Detected by Swift, Astrophys. J. 743 (2011) 62. 